\title{
Multicriteria Group Decision Making Using Fuzzy Approach for Evaluating Criteria of Electrician
}

\author{
Wiwien Hadikurniawati ${ }^{1}$, Khabib Mustofa ${ }^{2}$ \\ ${ }^{1}$ Faculty of Information Technology, Universitas Stikubank, Semarang, Indonesia \\ ${ }^{2}$ Department of Computer Science and Electronics, Universitas Gadjah Mada, Yogyakarta, Indonesia
}

\begin{tabular}{l} 
Article Info \\
\hline Article history: \\
Received Apr 20, 2016 \\
Revised Jul 19, 2016 \\
Accepted Jul 30, 2016 \\
\hline
\end{tabular}

Keyword:

Electrician

Fuzzy approach

Group decision making

Multicriteria

\begin{abstract}
This paper presents an approach of fuzzy multicriteria group decision making in determining alternatives to solve the selection problem of the electrician through a competency test. Fuzzy approach is used to determine the highest priority of alternative electrician who has knowledge and ability that best fits the given parameters. Linguistic variables are presented by triangular fuzzy numbers. They are used to represent a subjective assessment of the decisionmakers so that uncertainty and imprecision in the selection process can be minimized. Fuzzy approach require transforming crisp data to fuzzy numbers. Output of the best alternatives is generated by ranking method. Ranking has been made base on eight criteria which make the evaluation basis of each alternative. Ranking of the results is determined using different value of optimism index $(\alpha)$. The fuzzy multi criteria decision making (FMCDM) calculation is using the best alternative using three value of optimism index. The result of calculation shows that the same alternative reached from different index of optimism. This alternative is the highest priority of decision making process.
\end{abstract}

Copyright () 2016 Institute of Advanced Engineering and Science. All rights reserved.

\section{Corresponding Author:}

Wiwien Hadikurniawati,

Faculty of Information Technology,

Universitas Stikubank,

Trilomba Juang 1 Semarang.

Email: wiwien@edu.unisbank.ac.id

\section{INTRODUCTION}

Infrastructure development in Indonesia is rapidly growing. The effect of this development needs more elements of infrastructure development such as human resources. Worker as a human resources is one of the important elements that affects the survival and the implementation of construction projects. Improving the quality of human resources is very important because the construction industry needs experts in a huge numbers. Human resources competencies are a prerequisite for quality workers. Quality competence shows the ability or competency of worker as expected.

The government of Indonesia has recognized the importance of the human resources competency in the electricity enterprise so that the law of electricity are made in 1985. As the implementation of the legislation mentioned above, the government has developed a Government Regulation No. 3/2005 as an amendment to Government Regulation No. 10/1989 about the Provision and Use of Electric Power. It states that "Each engineer on the electricity business must have a certificate of competence".

To get a certificate of competence, an expert on electrical construction should do the several competency tests. Competency test consist of knowledge, skills and attitude tests. Knowledge, skill, and attitude tests obtained from several parts, both written and oral tests as well as practice of skill. Each component has many criteria. Competency test involves a number of assessors. To equate assessors's opinions of competency components tests used a fuzzy approach. Multicriteria decision making refers to the 
preferences of decision-making, such as evaluation, prioritization and selection of available alternatives [1]. Multi criteria decision making (MCDA) focuses on theoretical methodological development and practical applications of decision techniques to Decision Maker's evaluate alternatives [2].

Decision maker may consist more than one person or expert. Different background of each decision maker such as socio-culture, experience and intelligence sometimes make each decision maker gives difference preference. There can be different preferences of each criterion and preferences match rate each alternative on each criteria. Decision maker's opinion must be unified employing a unification process [3]. Multicriteria Decision making in groups decision makers can generate better solutions to complex problems involving the use of opinion of some experts. Decision problem occurred in many organizations. Some of these problems select a set of alternatives by considering multiple criteria. Cho [4] stated that the purpose of multicriteria decision making is reaching a decision by choosing the best alternative from several potential candidates, putting the subject to some criteria or attributes. The criteria or attribute can be the one causes some benefits or the one that causes cost.

$\mathrm{Fu}$ [5] stated, in order to achieve satisfaction in solving problems in a group decision making, group decision-making methods and model, the process of that usually consists of two processes, the agreement and the selection process.

Sudarma et.al [6] in his study discusses multi-criteria of decision making that has an alternative in the form of advice for students who will choose a course of study based on academic ability of students to pursue higher education. This study uses Elimination et Choix Tranduit La Realite (ELECTRE) method.

Christina [7] proposed the design of decision-making in uncertainty assessment approach using Analytical Hierarchy Process (AHP). The case study focused on a discrete decision. The preferences in this case use a key performance indicator. This process can solve the problem of consensus-based decisionmaking group to choose one of two options.

Low and Lin [1] in their research proposed a common fuzzy multi criteria decision making model, a concept fuzzy time weighted scheme. It adopted for considering in the model to build a fuzzy multiple criteria decision making with time weight (FMCDMTW). That model can take into account the time dependencies of the evaluation criteria and provide relativity low-cost way.

Daljooi and Eskandari [8] proposed model extracts regional contexts and visual topics from the image using multi criteria decision making approach based on Technique for Order of Preference by Similarity to Ideal Solution (TOPSIS) model. Mianabadi and Afshar [3] in their research stated that decision makers opinions were transformed into fuzzy preference relation and aggregated using OWA operator. This fuzzy group decision making algorithm was applied for a groundwater development problem.

Hegazy [9] explained how to solve the problem of uncertainty in the medical world, especially mental health issues using a fuzzy set approach. The uncertainties include assumptions of the experts and data. They are at risk due to uncertainties associated with medical condition. Chen et.al [10] and Dongjing [11] stated that the decision-making is a research group developed over the last twenty years, while research on the theory and method of group decision-making has always received attention from researchers in the world. Group decision-making is often difficult to determine the approval or agreement of the experts in giving preference because of differences in knowledge or judgment of each decision makers.

Tavana et.al [12] have been doing research on A hybrid fuzzy group decision support framework for assesment at NASA. The complicated structure of the assesment criteria and alternative are represented and evaluated with Analytical Network Process (ANP). The Alternative are ranked using fuzzy Technique for Order of Preference by Similarity to Ideal Solution (TOPSIS).

Wibowo [13] used a fuzzy multicriteria approach to the group decision making to increase the confidence level of the decision maker or the decision maker in solving the problem of selection of suppliers. Fuzzy linguistic variables are used to represent subjective assessment decision makers so that the uncertainty can be minimized.

Zhai et.al [14] stated that in complex system, are often faced with a decision problem that includes many attributes or many criteria and requires judgment or decision-making by some of the experts in a group. It requires consideration of the experts' judgments to resolve the uncertainty. Multicriteria decision making method can solve complex decision making problems, because: (a) the existence of a variety decision maker opinions, (b) the presence of uncertainty and imprecision, and (c) the decision making process is based on the concept of natural desire. The uncertainty and imprecision inherent in the problem of group decision making for specific alternatives must be chosen from several alternatives available, often conflict of criteria that involve repetitive decision makers. According to Tavana et.al [12], uncertainty and imprecision can make decision makers feel bad to give their subjective assessment because their positions are not fully confident in their assessments.

In this paper, selection of the best alternative from group decision makers is obtained using fuzzy multi attribute decision making methods. The proposed method use an approach of triangular fuzzy

Multicriteria Group Decision Making Using Fuzzy Approach for Evaluating .... (Wiwien Hadikurniawati) 
multicriteria group decision-making to solve decision makers's subjective assessment. Their judgments are represented by triangular fuzzy numbers specified in linguistic form.

Selection of the best alternative applied in the group decision-making problem to determine the competency test criteria for a qualified electrician. The rest of the paper is organised as follows. Section 2 decribes related work of our research. Section 3 explains about fuzzy multicriteria approach. Section 4 discusses a case study, analysis and result our research. Our conclusions are presented in Section 5.

\section{FUZZY MULTICRITERIA APPROACH}

Wibowo [13] stated that the selection of an appropriate alternative group decision-making includes several steps (a) determination of alternatives, (b) the determination of the selection criteria, (c) provision of rating the alternatives performance and weights of criteria, (d) aggregation of performance rating and weighted criteria to generate the overall performance index for all the alternatives and criteria, and (e) choosing the best alternative.

A major advantage of fuzzy logic is can be used as compensatory and non compensatory in a single model in different contexts, by using inferences through judgments provided by the Decision Maker (DM) [12].

To resolve the uncertainty and imprecision in fuzzy multicriteria group decision-making problems, the linguistic forms are used to facilitate the assessment of the decision makers. The linguistic forms are represented by triangular fuzzy numbers as their approximate value.

Fuzzy number is expressed as a fuzzy set a fuzzy interval in real number. The boundary of this interval is ambiguous; the interval is also a fuzzy set. Generally a fuzzy interval is represented by two end points $a_{1}$ and $a_{2}$ and a peak point $a_{2}$ as $\left[a_{1}, a_{2}, a_{3}\right]$ Triangular Fuzzy Numbers (TFN) is a fuzzy number represented by three values, namely $\mathrm{A}=(\mathrm{a}, \mathrm{b}, \mathrm{c})$. It can be defined as shown in equation (1).

$$
\mu_{(A)}(x)= \begin{cases}0, & x<a \\ \frac{x-a}{b-a}, & a \leq x \leq b \\ \frac{c-x}{c-b}, & b \leq x \leq c \\ 1, & x>c\end{cases}
$$

This presentation is expressed as membership functions as shown in the Figure 1.

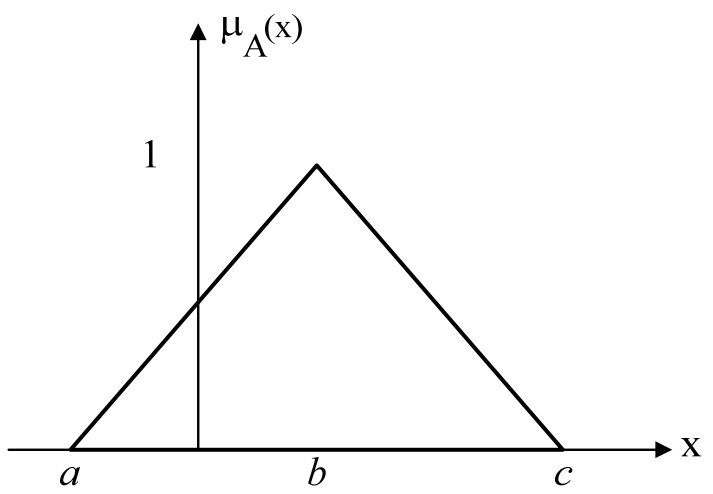

Figure 1.Triangular Fuzzy Number $A=(a, b, c)$

The evaluation and selection process start from each decision maker Dk $(\mathrm{k}=1,2, \ldots, \mathrm{k})$ gives the performance assessment (rating) for each decision alternative Ai ( $\mathrm{i}=1,2, \ldots, \mathrm{m}$ ) which is formed from $\mathrm{n}$ completion criteria $\mathrm{Cj}(\mathrm{j}=1,2, \ldots, \mathrm{n})$. The result is a decision matrix that contains the preferences of each decision-maker on any criteria expressed as equation (2):

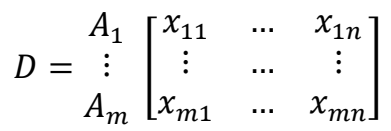


The weight vector $\mathrm{Wj}$ shows the influence of each criteria in decision making is expressed as:

$\mathrm{W}=\left[\begin{array}{llll}\mathrm{w}_{1} & \mathrm{w}_{2} & \ldots & \mathrm{w}_{\mathrm{n}}\end{array}\right]$

Let $\mathrm{w}_{\mathrm{ij}}=\left(\mathrm{a}_{\mathrm{ij}}, \mathrm{b}_{\mathrm{ij}}, \mathrm{c}_{\mathrm{ij}}\right), \mathrm{w}_{\mathrm{jk}} \in \mathrm{R}, \mathrm{j}=1,2, . ., \mathrm{n}, \mathrm{k}=1,2, . . \mathrm{k}$ is the weight given by the decision-makers $\mathrm{D}_{\mathrm{k}}$ of the criteria $\mathrm{Cj}$. Average weights $\mathrm{w}_{\mathrm{j}}^{\prime}=(\mathrm{a}, \mathrm{b}, \mathrm{c})$ of the criteria $\mathrm{Cj}$ is given by the decision-makers $\mathrm{k}(\mathrm{Dk})$ can be calculated by

$$
\mathrm{w}^{\prime}{ }_{\mathrm{j}}=(1 / \mathrm{k}) \otimes\left(\mathrm{w}_{\mathrm{j} 1} \oplus \mathrm{w}_{\mathrm{j} 2} \oplus \ldots \oplus \mathrm{w}_{\mathrm{jk}}\right)
$$

$\otimes$ and $\oplus$ are the operator of multiplication and addition on fuzzy numbers.

These weights (4) can be transformed by the equation (5):

$$
\mathrm{w}_{\mathrm{j}}^{\prime}=\frac{w j}{\sum_{j=1}^{n} w j}
$$

Fuzzy numbers of evaluations that have been carried out it is necessary to return the process defuzzyfication. It is change fuzzy numbers into crisp numbers. Numbers crisp be a single value. This is the final value obtained by weight calculation alternatives provided by each decision maker. The final value is the sum of the multiplication between weight and fitness index. The final value can be formulated as follows:

$$
r^{\prime}=\frac{1}{2}((\alpha c+b+(1-\alpha) a)
$$

$\mathrm{a}, \mathrm{b}$ and $\mathrm{c}$ are variables of triangular fuzzy number and $\alpha$ is an index of optimism that represents optimism level decision makers. The degree of optimism is in the range of $0 \leq \alpha \leq 1$. If the value of $\alpha$ is high, it indicates that decision makers have high optimism. To determine the rank of alternative, this formula can be used:

$$
S_{i}=\sum_{j=1}^{n} r^{\prime} i j
$$

Ranking of the alternatives is determined from the value of $S_{i}$. The greater value is the highest rank (more recommended by the decision maker).

\section{RESULTS AND ANALYSIS}

We use a fuzzy approach, the problem of determining the competency test criteria to evaluate and select a qualified electrician. The purpose of problem solving is to determine suitable criteria. Construction Services Agency is implementing competency test for experts in the field of electrical construction. In this competency test certification involves three assessors as decision makers. Problems to be solved is the determination of the level of importance of the criteria used in the competency test. Determination of the importance of this criterion will be used as the basis for a decision or judgment of a competent electrician. There are three alternative components of competence, S1 (knowledge), S2 (skill) and S3 (attitude). Each alternative is awakened from 8 criteria, C1 (written test 1), C2 (written test 2), C3 (written test 3), C4 (written test 4), C5 (written test 5), C6 (oral test), C7 (test of the theory of knowledge) and C8 (practice knowledge test). There are three assessors as decision maker D1, D2 and D3.

Linguistic value expressed by triangular fuzzy number is used to represent the preferences of decision makers so that the uncertainty and imprecision in the selection process can be minimized. The linguistic value of relative importance level or weight of each criteria are given by the respective decision makers. There are 5 linguistic forms of relative importance level, VL (Very Low), L (Low), M (Medium), H (High), VH (Very High). Each of them are represented by triangular fuzzy numbers.

$$
\begin{aligned}
& \mathrm{VL} \quad=(0,0.1,0.3) \\
& \mathrm{L}=(0.1,0.3,0.5) \\
& \mathrm{M}=(0.3,0.5,0.7) \\
& \mathrm{H}=(0.5,0.7,0.9) \\
& \mathrm{VH}=(0.7,0.9,1)
\end{aligned}
$$

The weight of each criteria for each decision maker can be shown in Table 1. 
Table 1. The weight of each criteria for each Decision Maker

\begin{tabular}{cccc}
\hline \multirow{2}{*}{ Criteria } & \multicolumn{3}{c}{ Decision Maker } \\
& D1 & D2 & D3 \\
\hline C1 & M & L & M \\
C2 & L & L & M \\
C3 & L & L & L \\
C4 & L & L & M \\
C5 & M & L & M \\
C6 & H & H & VH \\
C7 & H & H & VH \\
C8 & H & H & VH \\
\hline
\end{tabular}

The qualitative assessment given each decision-maker to each alternative rating of the competency test component represented by linguistic forms. They are VP (Very Poor), P (Poor), F (Fair), G (Good), VG (Very Good). They are represented by triangular fuzzy number as follows:

$\mathrm{VP}=(0,0,3)$

$\mathrm{P}=(0,3,5)$

$\mathrm{F} \quad=(2,5,8)$

$\mathrm{G}=(5,7,10)$

$\mathrm{VG}=(7,10,10)$

The rate of each alternative on each criteria for every decision maker is based on linguistic forms as shown in Table 2.

Table 2. Rating each alternative on each criteria by decision-maker

\begin{tabular}{ccccc}
\hline \multirow{2}{*}{ Criteria } & \multirow{2}{*}{ Alternative } & \multicolumn{3}{c}{ Decision Maker } \\
& & D1 & D2 & D3 \\
\hline \multirow{3}{*}{ C1 } & S1 & G & F & G \\
& S2 & F & F & F \\
& S3 2 & G & F & F \\
& S1 & G & F & G \\
& S2 & F & F & F \\
& S3 & G & F & F \\
& S1 & F & P & P \\
& S2 & F & P & P \\
& S3 & G & F & F \\
C4 & S1 & F & P & P \\
& S2 & F & P & P \\
& S3 & G & F & F \\
C5 & S1 & F & F & F \\
& S2 & F & F & F \\
& S3 & G & F & G \\
C6 & S1 & G & G & G \\
& S2 & G & G & G \\
& S3 & VG & G & G \\
C7 & S1 & G & G & VG \\
& S2 & G & G & VG \\
& S3 & VG & G & VG \\
& S1 8 & G & G & VG \\
& S2 & VG & G & VG \\
& S3 & VG & G & VG \\
\hline
\end{tabular}

The average weight for the first criteria (C1) can be calculated by using equation (5). The weight of criteria $1(\mathrm{C} 1)$ by decision maker 1 (DM1) is M (Medium), DM 2 is L (Low) and DM 3 is Medium (M). In accordance with the qualitative assesments of the decision maker, for $\mathrm{M}=(0.3,0.5,0.7)$ and $\mathrm{L}=(0.1,0.3$, $0.5)$, the average of criteria $\mathrm{C} 1$ as follows:

$$
\begin{aligned}
& w_{a_{1}}^{\prime}=\frac{0.3+0.1+0.3}{3}=0.2333 \\
& w_{b_{1}}^{\prime}=\frac{0.5+0.3+0.5}{3}=0.4333
\end{aligned}
$$




$$
w_{c_{1}}^{\prime}=\frac{0.7+0.5+0.7}{3}=0.6333
$$

The triangular fuzzy number for weight of $\mathrm{C} 1$ is $(0.2333,0.4333,0.6333)$. We use similar way to calculate the weight of another criteria (from C2 until C8). The results of average weight for each criteria is shown in Table 3.

Table 3. Average weight of each criteria

\begin{tabular}{ccccc}
\hline \multirow{2}{*}{ Criteria } & \multicolumn{3}{c}{ Decision Maker } & \multirow{2}{*}{ Average Weight $\left(\mathrm{w}_{\mathrm{j}}\right)$} \\
& $\mathrm{D} 1$ & $\mathrm{D} 2$ & $\mathrm{D} 3$ & \\
\hline C1 & $\mathrm{M}$ & $\mathrm{L}$ & $\mathrm{M}$ & $(0.2333,0.4333,0.6333)$ \\
C2 & $\mathrm{L}$ & $\mathrm{L}$ & $\mathrm{M}$ & $(0.1666,0.3666,0.5666)$ \\
C3 & $\mathrm{L}$ & $\mathrm{L}$ & $\mathrm{L}$ & $(0.1,0.3,0.5)$ \\
C4 & $\mathrm{L}$ & $\mathrm{L}$ & $\mathrm{M}$ & $(0.1666,0.3666,0.5666)$ \\
C5 & $\mathrm{M}$ & $\mathrm{L}$ & $\mathrm{M}$ & $(0,2333,0.4333,0.6333)$ \\
C6 & H & H & VH & $(0.5666,0.7666,0.9333)$ \\
C7 & H & H & VH & $(0.5666,0.7666,0.9333)$ \\
C8 & H & H & VH & $(0.6333,0.8333,0.9666)$ \\
\hline
\end{tabular}

The next step is calculating of average weight of each criteria based on decision makers preferences. Criteria 1 (C1) gives influence to alternative 1 (S1), S2 and S3. The preferences of decision maker (DM) 1 is $\mathrm{G}$ (Good), DM 2 is F (Fair) and DM 3 is $G$ (Good). $G=(3,7,10)$ dan $F=(2,5,8)$. The calculation of average weight of Criteria 1 toward each alternative $(\mathrm{S} 1, \mathrm{~S} 2, \mathrm{~S} 3)$ by each decision maker are:

$$
\begin{aligned}
& w_{c_{1} s_{1}}^{\prime}=\frac{5+2+5}{3}=4 \\
& {w^{\prime}{ }_{c_{1} s_{2}}}^{\prime}=\frac{7+5+7}{3}=6.3333 \\
& w_{c_{1} s_{3}}^{\prime}=\frac{10+8+10}{3}=9.3333
\end{aligned}
$$

\begin{tabular}{|c|c|c|c|c|c|}
\hline \multirow{2}{*}{ Criteria } & \multirow{2}{*}{ Alternative } & \multicolumn{3}{|c|}{ Decision Maker } & \multirow{2}{*}{ Average Weight $\left(x_{i j}\right)$} \\
\hline & & D1 & D2 & D3 & \\
\hline \multirow{3}{*}{$\mathrm{C} 1$} & S1 & $\mathrm{G}$ & $\mathrm{F}$ & $\mathrm{G}$ & $(4,6.3333,9.3333)$ \\
\hline & S2 & $\mathrm{F}$ & $\mathrm{F}$ & $\mathrm{F}$ & $(2,5,8)$ \\
\hline & S3 & G & $\mathrm{F}$ & $\mathrm{F}$ & $(3,5.6666,8.6666)$ \\
\hline \multirow{3}{*}{$\mathrm{C} 2$} & S1 & $\mathrm{G}$ & $\mathrm{F}$ & $\mathrm{G}$ & $(4,6.3333,9.3333)$ \\
\hline & S2 & $\mathrm{F}$ & $\mathrm{F}$ & $\mathrm{F}$ & $(2,5,8)$ \\
\hline & S3 & G & $\mathrm{F}$ & $\mathrm{F}$ & $(3,5.6666,8.6666)$ \\
\hline \multirow{3}{*}{$\mathrm{C} 3$} & S1 & $\mathrm{F}$ & $\mathrm{P}$ & $\mathrm{P}$ & $(0.6666,3.6666,6)$ \\
\hline & S2 & $\mathrm{F}$ & $\mathrm{P}$ & $\mathrm{P}$ & $(0.6666,3.6666,6)$ \\
\hline & S3 & G & $\mathrm{F}$ & $\mathrm{F}$ & $(3,5.6666,8.6666)$ \\
\hline \multirow{3}{*}{$\mathrm{C} 4$} & S1 & $\mathrm{F}$ & $\mathrm{P}$ & $\mathrm{P}$ & $(0.6666,3.6666,6)$ \\
\hline & S2 & $\mathrm{F}$ & $\mathrm{P}$ & $\mathrm{P}$ & $(0.6666,3.6666,6)$ \\
\hline & S3 & G & $\mathrm{F}$ & $\mathrm{F}$ & $(3,5.6666,8.6666)$ \\
\hline \multirow{3}{*}{$\mathrm{C} 5$} & S1 & $\mathrm{F}$ & $\mathrm{F}$ & $\mathrm{F}$ & $(2,5,8)$ \\
\hline & S2 & $\mathrm{F}$ & $\mathrm{F}$ & $\mathrm{F}$ & $(2,2,8)$ \\
\hline & S3 & G & $\mathrm{F}$ & G & $(4,6.3333,9.3333)$ \\
\hline \multirow{3}{*}{ C6 } & $\mathrm{S} 1$ & G & G & G & $(5,7,3.3333)$ \\
\hline & $\mathrm{S} 2$ & G & G & G & $(5,7,10)$ \\
\hline & S3 & VG & G & $\mathrm{G}$ & $(5.6666,8,10)$ \\
\hline \multirow{3}{*}{$\mathrm{C} 7$} & $\mathrm{~S} 1$ & $\mathrm{G}$ & G & VG & $(5.6666,8,10)$ \\
\hline & S2 & $\mathrm{G}$ & G & VG & $(5.6666,8,10)$ \\
\hline & $\mathrm{S} 3$ & VG & G & VG & $(6.3333,9,10)$ \\
\hline \multirow{3}{*}{$\mathrm{C} 8$} & $\mathrm{~S} 1$ & $\mathrm{G}$ & G & VG & $(5.6666,8,6.6666)$ \\
\hline & $\mathrm{S} 2$ & VG & G & VG & $(6.3333,9,10)$ \\
\hline & S3 & VG & G & VG & $(6.3333,9,10)$ \\
\hline
\end{tabular}

Results of the average rating of each alternative for each criteria are shown in Table 4.

Table 4. Average rating of each alternative for each criteria 
(C1).

Rating weighted for each alternative can be calculated as follows. For alternative 1 (S1), criteria 1

$$
r_{D M 1}=\left(\mathrm{w}_{\mathrm{S} 1} \cdot \mathrm{w}_{\mathrm{c} 1}\right) /\left(\Sigma \mathrm{w}_{\mathrm{S}} \cdot \Sigma \mathrm{w}_{\mathrm{DM} 1}\right)
$$

$r_{D M 1}=$ weighted rating criteria $(\mathrm{C} 1)$ for alternative $\mathrm{S} 1$ by DM1

$w_{S 1}=$ weighted average rating of alternative (S1) by DM1

$w_{c 1}=$ weighted average rating criteria $(\mathrm{C} 1)$ for alternative $(\mathrm{S} 1)$ by $\mathrm{DM} 1$

$\Sigma \mathrm{W}_{\mathrm{S}}=$ the amount of average weight for all alternatives by DM1

$\Sigma \mathrm{w}_{\mathrm{DM} 1}=$ the amount of average weight for all criteria by DM1

Calculation of a weighted rating for all alternatives shown in Table 5.

Table 5. Weighted rating for all alternatives

\begin{tabular}{ccccc}
\hline Alternative & Criteria & $\mathrm{r}_{\mathrm{D} 1}$ & $\mathrm{r}_{\mathrm{D} 2}$ & $\mathrm{r}_{\mathrm{D} 3}$ \\
\hline \multirow{4}{*}{$\mathrm{S} 1$} & 1 & 0.006261 & 0.037837 & 0.246316 \\
& 2 & 0.00313 & 0.029872 & 0.211129 \\
& 3 & 0.004696 & 0.033854 & 0.228721 \\
& 4 & 0.004471 & 0.032013 & 0.220374 \\
& 5 & 0.002235 & 0.025273 & 0.188893 \\
& 6 & 0.003353 & 0.028643 & 0.204632 \\
& 7 & 0.000563 & 0.019833 & 0.259659 \\
& 8 & 0.000563 & 0.019833 & 0.259659 \\
& 1 & 0.002532 & 0.030652 & 0.37506 \\
& 2 & 0.000937 & 0.024236 & 0.294246 \\
& 3 & 0.000937 & 0.024236 & 0.294246 \\
& 4 & 0.004218 & 0.037456 & 0.425018 \\
& 5 & 0.003213 & 0.038086 & 0.23752 \\
& 6 & 0.003213 & 0.015235 & 0.23752 \\
& 7 & 0.006425 & 0.048242 & 0.277106 \\
& 8 & 0.021178 & 0.057173 & 0.074475 \\
& 1 & 0.021178 & 0.057173 & 0.223428 \\
& 2 & 0.024002 & 0.065341 & 0.139635 \\
& 3 & 0.018668 & 0.0575 & 0.198135 \\
& 4 & 0.018668 & 0.0575 & 0.198135 \\
& 5 & 0.020864 & 0.064687 & 0.198135 \\
& 6 & 0.023474 & 0.060099 & 0.131827 \\
& 7 & 0.026236 & 0.067611 & 0.197742 \\
& 8 & 0.026236 & 0.067611 & 0.197742 \\
\hline
\end{tabular}

The next step is prioritizing an alternative decisions based on the results of aggregation. This priority is necessary to rank the decision alternatives. The results of the aggregation represented by using triangular fuzzy numbers. The final value is determined by using equation (6). This defuzzy method is used to determine ranks of alternatives, shown by equation 8 . The index of optimism $(\alpha)$ is used to solve this case is $\alpha=0, \alpha=0.5$ and $=1$. The index of optimism $(\alpha=1)$ shows not optimistic and $\alpha=0$ shows very optimistic. The result of calculation use index of optimism $(\alpha=0.5)$ as final value of alternative S1 (knowledge) shown in Table 6.

Table 6. Final Value of Alternative S1

\begin{tabular}{cccccccc}
\hline $\mathrm{r}_{1}{ }_{1}$ & $\mathrm{r}_{2}{ }_{2}$ & $\mathrm{r}_{3}{ }_{3}$ & $\mathrm{r}_{4}{ }_{4}$ & $\mathrm{r}_{5}{ }_{5}$ & $\mathrm{r}_{6}{ }_{6}$ & $\mathrm{r}_{7}{ }_{7}$ & $\mathrm{r}_{8}{ }_{8}$ \\
\hline 0.082063 & 0.072218 & 0.074972 & 0.085914 & 0.079226 & 0.0525 & 0.08295 & 0.068875 \\
\hline
\end{tabular}

To determine the rank of parameter on alternative S1 using equation (7) as follows:

$$
\begin{aligned}
& S 1=\sum_{j=1}^{8} r_{i j}= \\
& 0.082063+0.072218+0.074972+0.085914+0.079226+0.0525+0.082951+0.068875 \\
& =0.598718
\end{aligned}
$$

In the same formula, it can be calculated for alternative S2 (skill) and S3 (attitude). The results of complete calculations for all degree of optimism $(\alpha=0, \alpha=0.5$ and $\alpha=1)$ as shown in Table 7 . 
Table 7. Alternative Priority for Various Value $\alpha$

\begin{tabular}{cccc}
\hline Alternatives & $\alpha=0$ & $\alpha=0.5$ & $\alpha=1$ \\
\hline S1 & 0.2 .2771 & 0.598718 & 0.994666 \\
S2 & 0.186447 & 0.620095 & 1.053743 \\
S3 & 0.234407 & 0.722838 & 1.211269 \\
\hline
\end{tabular}

Table 7 shows that alternative 3 (S3) has the highest integral total value for each different index of optimism $(\alpha=0, \alpha=0.5$ and $\alpha=1)$. The selection results are obtained in the form of ranking the final value of criteria. Finally, the alternative S3 is the highest priority.

\section{CONCLUSION}

The process of evaluating and determining criteria in the competency test electricians is very complex and involves a group of decision-makers. There are many criteria and uncertainty and imprecision environment of decision making process. Our research develops an approach fuzzy multicriteria group decision-making to solve the problem of determining the competency test criteria. The case study shows that the fuzzy approach is used to solve the problems and the evaluation criteria used in determining the competency test expert electrical construction field. From the results with the triangular fuzzy approach attitude is the supreme criteria. This criteria must be considered by the assessor in determining a competent expert in the field of electricity. The result of the calculation fuzzy multi criteria decision making (FMCDM) is ultimately a recommendation for decision makers.

\section{REFERENCES}

[1] C. Y. Low and S. N. Lin, "Fuzzy Multiple Criteria Decision Making Model with Fuzzy Time Weight Scheme," TELKOMNIKA, vol/issue: 11(11), pp. 6831-6840, 2013.

[2] Y. Chen, et al., "A Decision Rule Aggregation Approach to Multiple Criteria Group Decision Support," 2008 International Conference on Systems, Man and Cybernetics IEEE, pp. 2514-2518, 2008.

[3] H. Mianabadi and A. Afshar, "Fuzzy Group Decision Making to Select the Best Alternative for Development of Groundwater Resources," Sixth International Conference on Fuzzy Systems and Knowledge Discovery, DOI. 10.1109/FSKD.2009.845, IEEE, pp. 307-311, 2009.

[4] K. T. Cho, "Multicriteria Decision Methods: An Attempt to Evaluate and Unify," Mathematical and Computer Modelling, Elsevier, pp. 1099-1119, 2003.

[5] C. Fu, "A Consensus Framework for Multiple Attribute Group Decison Analysis in Evidential Reasoning Context," Information Fusion, DOI. 10.1016/j.inffus.2011.12.002, Elsevier B.V, pp. 22-35, 2011.

[6] M. Sudarma, et al., "Decision Support System for the Selection of Courses in the Higher Education using the Method of Elimination Ef Choix Tranduit La Realite," International Journal of Electrical and Computer Engineering (IJECE), vol/issue: 5(1), pp. 129-135, 2015.

[7] J. H. Christina, et al., "Multi-Criteria Decision Making Uncertainty in Building Performance Assesment," Building and Environment, DOI 10.1016/j.buildenv.2013.07.019, Elsevier, pp. 81-90, 2013.

[8] H. Daljooi and A. R. Eskandari, "A Novel Semantic Statistical Model for Automatic Image Annotation Using the Relationship between the Regions Based on Multi Criteria Decision Making," International Journal of Electrical and Computer Engineering (IJECE), vol/issue: 4(1), pp. 37-51, 2014.

[9] S. Hegazy, et al., "Modulating Membership Grades to Gain Consensus for Fuzzy Set Uncertainty Values in Clinical Decision Support System", Third International Conference on Advances in Human-Oriented and Personalized Mechanisms, Technologies and Services, IEEE, pp. 40-45, 2010.

[10] X. Chen, et al., "An Analytic Method far Consensus Analyisis in Group Experts'Judgments Based on Numerical Decision Matrix Preference Information," Fifth International Conference on Fuzzy Systems and Knowledge Discovery, DOI. 10.1109/FSKD.2008.502. IEEE, pp. 176-180, 2008.

[11] P. Dongjing, "A New Method for Fuzzy Multiple Attribute Decision Making based on Interval Numbers," International Forum on Information Technology and Applications, DOI 10.1109/IFITA.2009.141, IEEE, pp. 408$411,2009$.

[12] M. Tavana, et al., “A Fuzzy multi-criteria Decision Analysis Model for Advanced Technology Assesment at Kennedy Space Center,” Journal Operational Research Soc, DOI. 10.1057/jors.2009.107, vol. 61, pp. 1459-1470, 2010.

[13] S. Wibowo, et al., "A Fuzzy Multicriteria Group Decision Making Approach for Improving the Degree of Confidence in Supplier Selection," Fourth International Symposium on Computational Intelligence and Design, DOI 001.10.1109/ISCID.2011.122. IEEE, pp. 83-86, 2011.

[14] X. Zhai, et al., "A Multiple Criteria Decision Method Based on Uncertain Judgment," Sixth International Conference on Fuzzy Systems and Knowledge Discovery, pp. 218-222, 2009. 